\title{
Path Dependent Coordination of Expectations in Asset Pricing Experiments: a Behavioral Explanation
}

\author{
Anna Agliari*1 , Cars H. Hommes ${ }^{\dagger 2}$, Nicolò Pecora ${ }^{\ddagger 1}$ \\ ${ }^{1}$ Dept. of Economics and Social Science, Catholic University, Piacenza, Italy \\ ${ }^{2}$ CeNDEF, Amsterdam School of Economics, University of Amsterdam and Tinbergen Institute, \\ Netherlands
}

September 26, 2015

\begin{abstract}
In the learning-to-forecast laboratory experiments in Hommes et al. (2005), three different types of aggregate asset price behavior have been observed: monotonic convergence to the stable fundamental steady state, dampened price oscillations and permanent price oscillations. We present a simple behavioral 2-type heuristics switching model explaining individual as well as aggregate behavior in the experiment. Based on relative performance, agents switch between a simple trend-following and an anchor and adjustment heuristic that differ in how much weight is given to the long run average price level. The nonlinear switching model exhibits path dependence through co-existence of a locally stable fundamental steady state and a stable (quasi-)periodic orbit, created via a so-called Chenciner bifurcation. Depending on initial states, agents coordinate individual expectations either on a stable fundamental steady state path or on almost self-fulfilling persistent price fluctuations around the fundamental steady state.
\end{abstract}

Keywords: multi-agent systems; asset pricing model; experimental economics; coexistence of attractors; expectation feedback.

JEL codes: E32, G12, D84, C91, C62

Acknowledgment: Financial support from the EU $7^{\text {th }}$ framework collaborative projects "MacroRisk Assessment and Stabilization Policies with New Early Warning Signals (RAStaNEWS)", grant no 320278 and "Integrated Macro-Financial Modelling for Robust Policy Design (MACFINROBODS)", grant no 612796, and from the Netherlands Institute of Advanced Studies in the Humanities and Social Sciences (NIAS) is gratefully acknowledged. None of the above are responsible for errors in this paper.

${ }^{*}$ Corresponding author: Via Emilia Parmense 84, 29100, Piacenza. anna.agliari@unicatt.it

${ }^{\dagger}$ Roeterstraat 11, NL-1018 WB Amsterdam. C.H.Hommes@uva.nl

${ }^{\ddagger}$ Via Emilia Parmense 84, 29100, Piacenza. nicolo.pecora@unicatt.it 


\section{Introduction}

There are many historical examples of asset or commodity market bubbles, with excessive growth of prices followed by sudden collapse. One of the first and perhaps the most famous is the Dutch "tulipmania" in 1636-1637, when tulip bulbs hit price levels equivalent to several average yearly wage salaries, before they suddenly collapsed in February 1637 (Kindleberger, 2001). More recent examples include the "dot-com" bubble in the high tech NASDAQ stock market with its peak in May 2000 and the housing price bubbles in the U.S. and many other countries between 2000 and 2010. A bubble is defined as a strong and persistent overvaluation of an asset compared to its economic "fundamental value". Large bubbles and sudden market crashes are hard to reconcile with the standard rational expectations representative agent model, which typically assumes that prices track fundamental value. Popular explanations of the occurrence of bubbles often rely on some form of Greenspan's "irrational exuberance" as an amplification mechanism after (small) fundamental shocks (e.g. Shiller, 2000).

Behavioral finance has documented an increasing list of mechanisms emphasizing the role of investor psychology as an amplification mechanism in explaining large asset price movements, e.g. overconfidence, wishful thinking, gambler's fallacy, momentum trading, trend extrapolation, belief anchoring, availability heuristics, reference dependent utility, loss aversion, ambiguity aversion, etc. (Barberis and Thaler, 2003).

Despite their popularity in the financial press, the existence and empirical relevance of financial bubbles and crashes is still heavily debated among academic economists. The most important reason for the controversy may be that the "fundamental value" of an asset is difficult to measure in real markets and depends e.g. on assumptions about preferences and risk premia. Experiments in a controlled laboratory environment are therefore an important complementary tool to gain insights into possible causes and circumstances that may lead to significant and persistent price deviations from a controlled fundamental value. A seminal contribution are the bubble experiments in Smith et al. (1988). Subjects can buy an asset that pays a dividend 1 each period. The market lasts 15 periods, so that fundamental value is a decreasing step function from 15 to 0 . Experimental markets typically do not track the fundamental, however, but rather start below fundamental with a price around $4-5$, after which the price starts to increase, then becomes overvalued reaching a maximum up to 15 or more around period 10, and finally collapses to 0 towards the end of the experiment. There is a large literature showing that these experimental asset market bubble and crashes are robust w.r.t. many variations in the experimental design (see Palan, 2013 for an extensive survey $)^{1}$.

\footnotetext{
${ }^{1}$ Kirchler et al. (2012) and Huber et al. (2012) show however that the bubbles disappear when a more accurate framing of the fundamental value is used by describing it as the depletion of a gold mine rather than a stock market. Dufwenberg et al. (2005) studied repeated bubble experiments with experienced and inexperienced traders and show that bubbles disappear when (part of) the subjects become more experienced. These results show that bubbles in experimental markets are still not fully understood and more experiments are needed to shed light on the circumstances in which bubbles may or may not prevail.
} 
To study the role of expectations in generating bubbles and crashes, Hommes et al. (2005) ran so-called learning-to-forecast asset pricing experiments with a constant fundamental value and computerized optimal trading. Subjects play the role of professional forecasters and are asked to submit point forecasts for the price of a risky asset for 50 periods. The risky asset pays an uncertain dividend in each period. Individual forecasts feed into a standard mean-variance demand function and the price of the risky asset, $p_{t}$, is determined every period by market clearing, as an aggregation of individual forecasts of all participants. An important feature of these experimental asset markets is the positive feedback, that is, the higher the individual forecasts, the larger the demand for the risky asset and the higher the realized market price. The fundamental price of the risky asset is not explicitly given to the subjects, but can be computed as the discounted sum of expected future dividends from common knowledge of the mean dividend $\bar{y}$ and the risk-free interest rate $r$. In the experiment, the fundamental price becomes $p^{f}=\bar{y} / r=60$.

In 20 experimental markets, three different patterns of aggregate behavior have been observed (Figure 1a-c): (i) slow and almost monotonic convergence to the fundamental price, (ii) persistent oscillations around the fundamental value and (iii) dampened price oscillations. Moreover, participants are able to coordinate on a common almost selffulfilling forecasting strategy, but this strategy can be different between groups. The analysis of individual prediction strategies in Hommes et al. (2005) reveals that the dispersion between prediction strategies is much smaller than the forecast errors participants make on average. This indicates that participants within a group coordinate on a common prediction strategy. Although participants make forecasting errors, they are similar in the way that they make these errors. Estimation of the individual prediction strategies shows that participants tend to use simple linear prediction strategies, such as naive expectations, adaptive expectations or "autoregressive" expectations. Again, participants within a group coordinate on using the same type of simple prediction strategy. These prediction strategies make relatively small errors and are in this sense almost selffulfilling. Almost self-fulfilling equilibria are a key feature of positive feedback systems, in particular when they are near-unit root systems (see Hommes (2013a) for a discussion). Since the discount factor $1 /(1+r)$ is very close to 1 , the price generating mechanism of the asset pricing experiment (see Eq. 1) follows a near-unit root process. If the system would have a unit root, a continuum of rational expectations steady states would exist. Since the system is near unit root, it has a continuum of almost self-fulfilling equilibria. What the lab experiments in Hommes et al. (2005) then have shown is that agents may coordinate on an oscillatory pattern or sequence of almost self-fulfilling equilibria.

The purpose of this paper is to develop a simple 2-type behavioral heuristics switching model which is able to explain the path-dependent coordination of individual expectations on these different observed aggregate outcomes. Agents are boundedly rational and switch between two simple forecasting heuristics based on their relative performance. Strategy switching is thus based on an evolutionary selection mechanism. The two forecasting heuristics are a simple trend-following rule and an anchor and adjustment heuristic. Both rules extrapolate the latest observed price trend, but differ in their 
anchor describing how much weight is given to the last price observation and to the long run average price level. The nonlinear switching model exhibits path dependence through co-existence of a locally stable fundamental steady state and a stable (quasi-)periodic orbit. Depending on initial states, agents coordinate individual expectations either on a locally stable fundamental steady state path or on persistent (quasi-)periodic price fluctuations around the fundamental steady state. The fundamental steady state is the homogeneous rational expectations outcome, while the persistent fluctuations around the steady state are not perfect foresight solutions, but nevertheless are almost self-fulfilling. We illustrate these intuitive results by model simulations, but also rigorously establish path-dependence and co-existence of a locally stable fundamental steady state and stable periodic or quasi-periodic orbits by mathematical bifurcation analysis as a generic feature of our nonlinear behavioral switching model. We find that our simple model exhibits a so-called Chenciner bifurcation explaining the creation of a co-existing stable steady state and a quasi-periodic attractor. This simple mechanism may explain why markets are path-dependent and may exhibit temporary bubble and crash dynamics.

Our model builds on the adapted belief system (ABS) introduced by Brock and Hommes $(1997,1998)$. In an ABS boundedly rational agents choose from a pool of forecasting rules and learn through evolutionary selection or reinforcement learning based upon relative performance. The main behavioral learning mechanism thus dictates that agents gradually switch to better performing rules. Anufriev and Hommes (2012a) used an ABS with 4 different forecasting rules (adaptive expectations, two trend-following rules and an anchor and adjustment rule) to explain individual and aggregate behavior in the asset pricing laboratory experiments in Hommes et al. (2005). Their analysis is based on stochastic simulations and path-dependence only occur due to (small) exogenous shocks. In the absence of external stochastic shocks, the 4-type model has a globally stable fundamental steady state (Anufriev and Hommes, 2012b). In contrast, our simpler 2-type behavioral model exhibits endogenous path-dependence, with co-existence of a locally stable steady state and stable (quasi-)periodic attractor even in the absence of any exogenous shocks.

The heterogeneous expectations models also bear similarities with the noise trader literature (DeLong et. al., 1990). In the noise trader models one of the agents is assumed to be perfectly rational, taking into account the presence and actions of non-rational traders. In the ABS in Brock and Hommes (1998) typically all agents are boundedly rational and are unable to perfectly foresee the presence, the beliefs and the actions of non-rational traders ${ }^{2}$. Our simple 2-type model thus provides a behavioral explanation of individual coordination and aggregate behavior. This approach fits well into the behavioral agent-based modeling approach in finance (Arthur, 1993; Farmer and Foley, 2009; Hommes, 2006; LeBaron, 2006).

The paper is organized as follows: Section 2 briefly describes laboratory experiments

\footnotetext{
${ }^{2}$ Brock and Hommes (1997) introduced heterogeneous expectations in a cobweb model with perfectly rational versus simple naive expectations, similar to the noise trader approach. Brock and Hommes, however, assign information costs to rational expectations and, most importantly, the fractions of both agent types evolves endogenously over time driven by performance based evolutionary selection.
} 
with human subjects; in Section 3 the 2-type heuristics switching model is presented. Section 4 is devoted to the stability analysis of the steady state of the model, while simulations are performed in Section 5 and Section 6 concludes. An appendix presents the mathematical bifurcation analysis of the model.

\section{Laboratory experiments}

Hommes et al. (2005) performed a computerized learning to forecast asset pricing experiments in the CREED laboratory at the University of Amsterdam. In each session 6 participants play the role of advisors, professional forecasters, to large pension funds and have to submit point forecasts for the price of a risky asset for 50 consecutive periods. The pension fund can invest either in a risk-free asset with real interest rate $r$ per period or in shares of an infinitely lived risky asset paying each period an uncertain dividend, which is an independent and identically distributed random variable with mean $\bar{y}$. The price of the risky asset, $p_{t}$, is determined every period by a market clearing equation, as an aggregation of individual forecasts of all participants. The exact functional form of the market equilibrium equation was unknown to the participants, but they were informed that the market exhibits positive feedback, that is, the higher their forecasts, the larger the demand of the pension fund for the risky asset and the higher the realized market price. Participants also know the values of the risk-free interest rate $r=0.05$ and the mean dividend $\bar{y}=3$, and therefore they have enough information to compute the rational fundamental price of the risky asset $p^{f}=\bar{y} / r=60$. This however only happened rarely ${ }^{3}$.

Every session of the experiment lasts 51 periods and in every period each of the six participants provide a two-period-ahead forecast for the price of the risky asset, given the available information which consists of past prices (up to two lags) of the risky asset and own past predictions (up to one lag) made by the participant. The predictions of other participants are unknown. When all six predictions for the price in period $t+1$ are submitted, the market clearing price is computed, using a standard asset pricing model with mean-variance demand and heterogeneous beliefs (Hommes et al., 2005; Brock and Hommes, 1998):

$$
p_{t}=\frac{1}{1+r}\left(\left(1-n_{t}\right) \bar{p}_{t+1}^{e}+n_{t} p^{f}+\bar{y}+\varepsilon_{t}\right), \quad t=0, \ldots, 50
$$

where $\bar{p}_{t+1}^{e}=\frac{1}{6} \sum_{i=1}^{6} p_{i, t+1}^{e}$ is the (equally weighted) average of the six individual forecasts, $r$ is the risk-free interest rate, $\bar{y}$ is the mean dividend, $\varepsilon_{t}$ is a (small) stochastic term representing small demand/supply shocks and $n_{t}$ stands for a small fraction of "robot" traders who always submit a fundamental forecast $p^{f}$. Robot traders were introduced as a far from equilibrium stabilizing force in the experiment to prevent the occurrence

\footnotetext{
${ }^{3}$ Hommes et al. (2005) report one subject whose forecast is $p^{e}=p^{f}=60$ in the first four periods. Because all other subjects forecast lower prices, however, this subject changed his forecasting strategy after four periods.
} 
of large bubbles. The fraction of robot traders increased as the price moved away form its fundamental equilibrium level, according to ${ }^{4}$

$$
n_{t}=1-\exp \left(-\frac{1}{200}\left|p_{t-1}-p^{f}\right|\right)
$$

Since prices are restricted to the interval $[0,100]$, the fraction of fundamental robot traders never exceeds 0.25 . At the end of each period every participant $h$ was informed about the realized price and his/her earnings were defined by a quadratic scoring rule ${ }^{5}$ :

$$
e_{t, h}=\operatorname{Max}\left(1300-\frac{1300}{49}\left(p_{t}-p_{t, h}^{e}\right)^{2}, 0\right)
$$

The main results of the experiments may be summarized as follows (Figure 1):

- human subjects tend to follow simple forecasting rules (heuristics), based upon recent past observations, such as trend-following and anchor and adjustment rules;

- participants are able to coordinate on a common almost self-fulfilling prediction strategy, but this strategy can be different between sessions;

- realized asset prices are significantly different from the homogeneous rational fundamental price in all sessions.

- the system exhibits path dependence as across 20 markets three different price patterns were observed (Figure 1a-c): slow and almost monotonic convergence, persistent oscillations with almost constant amplitude, dampened oscillations.

\section{Two type heuristics switching model}

The general setup of the evolutionary selection or heuristics switching model follows Brock and Hommes (1997) and Anufriev and Hommes (2012a). There exists a pool of simple forecasting rules (e.g. adaptive or trend-following heuristics) commonly available to the agents, who select rules from this pool. At every time period these heuristics give forecasts for next period's price, and the realized market price is an aggregation of these individual forecasts. Moreover the fractions of agents using different forecasting heuristics are changing over times, because individuals are learning based on evolutionary selection: the better a forecasting rule performed in the recent past, the higher will be the fraction of agents adopting that rule. Hence, the realized market price and the fractions of the forecasting heuristics co-evolve in a dynamic process with mutual feedback.

\footnotetext{
${ }^{4}$ Similar experiments without fundamental robot traders, resulting in much more unstable price behavior characterized by large bubbles and crashes, have been done in Hommes et al. (2008).

${ }^{5}$ In the learning-to-forecast experiments subjects were paid by forecasting performance. Bao et al. (2014) recently ran learning-to-forecast and learning-to-optimize experiments, where subjects not only forecast but also engage in trading activities and are (partly) paid by realized profits or utility. In these learning-to-optimize experiments similar results with price oscillations around the fundamental, bubbles and market crashes are obtained.
} 


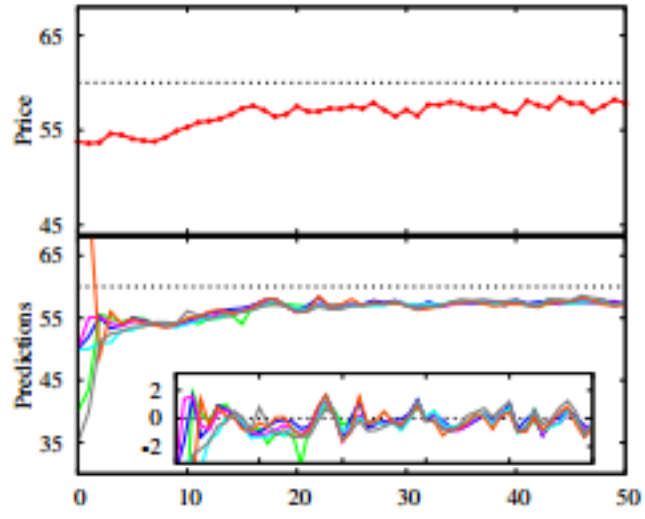

(a) (Almost) monotonic convergence

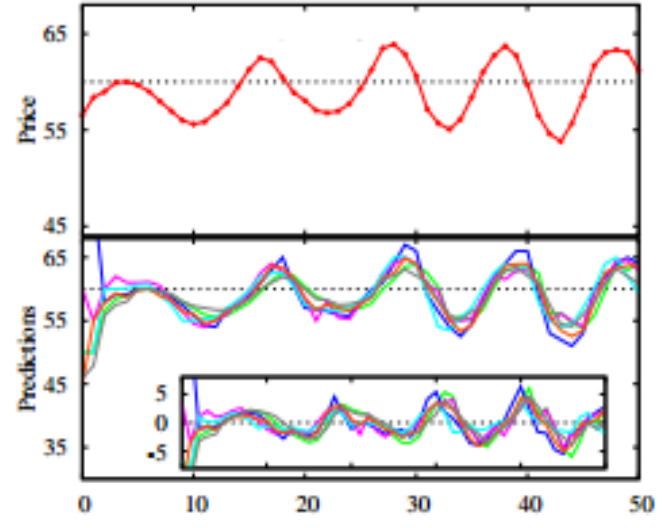

(b) Persistent oscillations

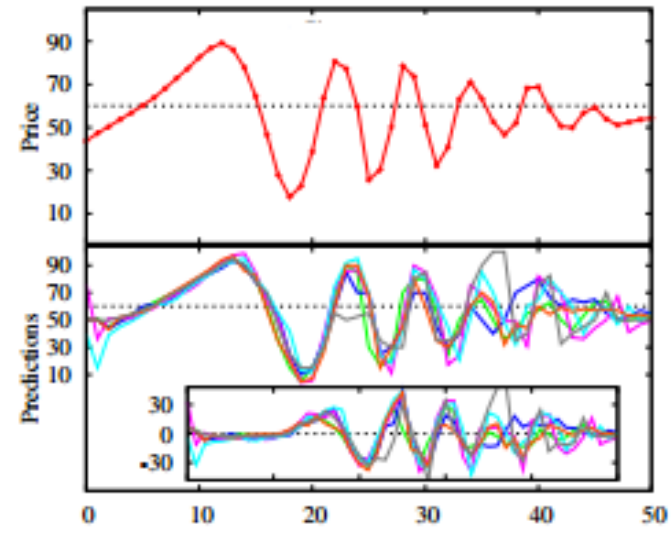

(c) Dampening fluctuations

Figure 1: Learning-to-forecast laboratory experiments (Hommes et al., 2005). Price time series (upper part), individual predictions of 6 participants (lower part) and forecasting errors (inner frame) of laboratory experiments. In 20 markets three different types of aggregate behavior were observed (Figure 1a-c): almost monotonic convergence, persistent oscillations with almost constant amplitude, dampened oscillations; 
Let $\mathfrak{I}$ denote a set of $I$ heuristics which agents can use for price prediction. In the beginning of period $t$ every rule $i \in \mathfrak{I}$ gives a two period-ahead point prediction for the price $p_{t+1}$. This prediction is described by a deterministic function $f_{i}$ of the available information set:

$$
p_{i, t+1}^{e}=f_{i}\left(p_{t-1}, p_{t-2}, \ldots ; p_{i, t}^{e}, p_{i, t-1}^{e}, \ldots\right) .
$$

Notice that (4) is a 2-period ahead forecasting rule as $p_{t}$ is unknown yet when the individual forecasts $p_{i, t+1}^{e}$ are formed. The price in period $t$ is computed on the basis of these predictions, and it is given by the market clearing price equation (1) of the previous section (we recall it for convenience):

$$
p_{t}=\frac{1}{1+r}\left(\left(1-n_{t}\right) \bar{p}_{t+1}^{e}+n_{t} p^{f}+\bar{y}+\varepsilon_{t}\right)
$$

where $\bar{p}_{t+1}^{e}$ denotes the average of the individual forecasts, $p^{f}$ is the rational fundamental price, $r(=0.05)$ is the risk free interest rate, $\bar{y}(=3)$ is the mean dividend, $\varepsilon_{t}$ is the stochastic term associated with small demand/supply shocks and $n_{t}$ represents the timevarying share of fundamental robot traders as described by Equation (2). Recall that the fraction of robot traders increases in response to the deviations of the asset price from its fundamental level according to (2) and that the fundamental price is set to $p^{f}=\bar{y} / r=3 / 0.05=60$.

The average $\bar{p}_{t+1}^{e}$ in (5) is a population weighted average of the different forecasting rules:

$$
\bar{p}_{t+1}^{e}=\sum_{i=1}^{I} n_{i, t} p_{i, t+1}^{e} .
$$

The fraction of each heuristic, $n_{i, t}$, evolves over time and depends on the past relative performance, with more successful heuristics attracting more followers. The performance measure of a forecasting heuristic is based on its squared forecasting error. More precisely, the performance measure of heuristic $i$ up to (and including) time $t-1$ is

$$
U_{i, t-1}=-\left(p_{t-1}-p_{i, t-1}^{e}\right)^{2}+\eta U_{i, t-2} .
$$

The parameter $\eta$ represents the memory of the agents, measuring the relative weight that each agent gives to past forecasting errors. If $\eta=0$, the impact of each heuristic is completely determined by the most recent forecast error; for $0<\eta \leq 1$ all past prediction errors affect the fraction of heuristic $i$, with exponentially declining weights. Given the performance measure, the fraction of agents using heuristic $i$ is updated according to a discrete choice model with a-synchronous updating (Hommes et al. 2005 and Diks et al. 2005):

$$
n_{i, t}=\delta n_{i, t-1}+(1-\delta) \frac{\exp \left(\beta U_{i, t-1}\right)}{Z_{t-1}}
$$


where $Z_{t-1}=\sum_{i=1}^{I} \exp \left(\beta U_{i, t-1}\right)$ is a normalization factor so that $\sum_{i=1}^{I} n_{i, t}=1$. If $\delta=0$ the updating rule reduces to the discrete choice model with synchronous updating. The more general case $0<\delta<1$, gives some persistence in the fraction of rule $i$, reflecting the fact that not all participants update their rule in every period or at the same time. For this reason $\delta$ can be seen as the average per period fraction of agents who stick to previous forecasting rule. Hence, for $0<\delta<1$ in each period a fraction $1-\delta$ of individuals update their rule according to the well known discrete choice model, used for example in Brock and Hommes (1997). $\quad \beta$ represents the intensity of choice and measures how sensitive agents are to differences in strategy performance: the higher $\beta$, the faster individuals will switch to more successful rules. For $\beta=0$, the result is an equal distribution of forecasting rules among individuals; in the other extreme case $\beta \rightarrow \infty$, the fraction $1-\delta$ who updates its heuristic switches immediately to the most successful predictor.

To keep the model as simple as possible, but rich enough to explain the different aggregate price patterns observed in the experiments, we select only two forecasting heuristics. These simple rules are among the ones estimated on the individual forecasts in the experiments. A behavioral interpretation underlies each heuristic.

The first heuristic is a simple trend following rule (TR). It extrapolates a trend that can be weak or strong depending on what value is assigned to the parameter $g$ :

$$
p_{1, t+1}^{e}=p_{t-1}+g\left(p_{t-1}-p_{t-2}\right) .
$$

This rule means that agents predict the last observed price level plus a multiple of the last observed price change $(g>0)$. The 4-type heuristics switching model of Anufriev and Hommes (2012a, 2012b) had two trend-following rules, a weak and a strong trendfollowing rule with coefficients $g=0.4$ and $g=1.3$.

The second rule is slightly more sophisticated. First consider a trend-following rule that uses a more sophisticated anchor, a weighted average of the long-run time average of historical prices $p_{t-1}^{a v}$ and the last observed price $p_{t-1}$ :

$$
p_{t+1}^{e}=(1-m) p_{t-1}^{a v}+m p_{t-1}+l\left(p_{t-1}-p_{t-2}\right),
$$

where $p_{t-1}^{a v}$ is the sample average of all past prices, i.e. $p_{t-1}^{a v}=\frac{1}{t} \sum_{j=0}^{t-1} p_{j}$. Following Kahnemann and Tversky (1973), Tversky and Kahnemann (1974), this rule is called learning anchoring and adjustment heuristic (LAA), since it uses an anchor $m p_{t-1}^{a v}+$ $(1-m) p_{t-1}$, defined as a weighted average between the last observed price and the sample mean of all past prices, and extrapolates the last price change $\left(p_{t-1}-p_{t-2}\right)$ by a factor $l$. The parameter $m$ represents the weight assigned to the last observed price, while $1-m$ is the weight assigned to the long run sample average. In the 4-type heuristics switching model of Anufriev and Hommes (2012a) an anchor and adjustment rule with coefficients $m=0.5$ and $l=1$ is used.

Including the sample average $p_{t-1}^{a v}$ of all past prices in one of the forecasting rules would lead to a dynamic model of very high dimension. Therefore, we use simpler, but closely related anchoring and adjustment heuristic (A\&A):

$$
p_{2, t+1}^{e}=(1-m) p^{f}+m p_{t-1}+l\left(p_{t-1}-p_{t-2}\right),
$$


where the sample average $p_{t-1}^{a v}$ has been replaced by the fundamental price level $p^{f}$. The rule (11) is a simple $\mathrm{AR}(2)$ rule and such rules have successfully been estimated, with almost the same coefficients, $m \approx 0.5$ and $l \approx 1$, for a number of subjects in Hommes et al. (2005). For tractability we use the anchor and adjustment rule (11) as our second forecasting rule, as an approximation of (10).

From a behavioral perspective, there is an important difference between the trend following rules in (9) and (10-11). The pure trend extrapolation rule (9), with the last observed price $p_{t-1}$ as its anchor, always predicts an increase of the price after an observed increase in the last period. In contrast, with an anchor giving more weight to the observed fundamental equilibrium level of prices, rules (10) or (11) are able to predict turning points of observed price fluctuations when the price moves away from its long run equilibrium level. Anufriev and Hommes (2012a) explained the observed permanent price oscillations in the experiment through coordination on the LAA rule in their 4-type heuristics switching model simulations.

All these rules are first order heuristics in the sense that they only use the last observation, i.e. the last price, the last forecast and the last price change. Both rules (9) and (11) can be collected into one general rule with two lags:

$$
p_{i, t+1}=\left(1-\beta_{i, 1}-\beta_{i, 2}\right) p^{f}+\beta_{i, 1} p_{t-1}+\beta_{i, 2} p_{t-2}, \quad i=1,2 .
$$

The trend extrapolating rule (9) is obtained for $\beta_{1,1}=1+g$ and $\beta_{1,2}=-g$, whereas the anchoring and adjusting rule (11) is obtained setting $\beta_{2,1}=m+l$ and $\beta_{2,2}=-l$.

To understand the dynamical behavior, it is important to consider the deterministic skeleton of the model, where we fix the noise $\varepsilon_{t} \equiv 0$ in the pricing equation (5):

$$
\left\{\begin{array}{l}
p_{1, t+1}^{e}=p_{t-1}+g\left(p_{t-1}-p_{t-2}\right) \\
p_{2, t+1}^{e}=(1-m) p^{f}+m p_{t-1}+l\left(p_{t-1}-p_{t-2}\right) \\
n_{t}=1-\exp \left(-\frac{1}{200}\left|p_{t-1}-p^{f}\right|\right) \\
U_{i, t-1}=-\left(p_{t-1}-p_{i, t-1}^{e}\right)^{2}+\eta U_{i, t-2} \quad i=1,2 \\
n_{i, t}=\delta n_{i, t-1}+(1-\delta) \frac{\exp \left(\beta U_{i, t-1}\right)}{Z_{t-1}} \quad i=1,2 \\
p_{t}=\frac{1}{1+r}\left(\left(1-n_{t}\right)\left(n_{1, t} p_{1, t+1}^{e}+n_{2, t} p_{2, t+1}^{e}\right)+n_{t} p^{f}+\bar{y}\right)
\end{array}\right.
$$

We will show that this simple nonlinear switching model with 2-types can explain the three types of observed aggregate behavior in Figure 1 for suitable and empirically relevant parameter values. Moreover, for a given, fixed set of empirically relevant parameter values, the 2-type model can explain path dependent coordination of expectations on either a stable fundamental steady state or persistent fluctuations around the fundamental steady state. 


\section{Stability Analysis of Fundamental Steady State}

The dynamics described by (13) can be re-written in deviations from the fundamental price, setting

$$
x_{1, t}=p_{t}-p^{f}, \quad x_{2, t}=x_{1, t-1}, \quad x_{3, t}=x_{1, t-2}, \quad x_{4, t}=x_{1, t-3}
$$

and plugging the two heuristics (9) and (11) into the price equation (5). This leads to the following 7-dimensional system consisting of two equations describing the evolution of performance measures $U_{1, t-1}$ and $U_{2, t-1}$, one describing the fraction $n_{1, t}$ of the first forecasting heuristic, one equation describing the price dynamics and three more equations used to take lagged price deviations into account:

$$
\left\{\begin{array}{l}
U_{1, t-1}=\eta U_{1, t-2}-\left(x_{1, t-1}-(1+g) x_{3, t-1}+g \cdot x_{4, t-1}\right)^{2} \\
U_{2, t-1}=\eta U_{2, t-2}-\left(x_{1, t-1}-(m+l) x_{3, t-1}+l \cdot x_{4, t-1}\right)^{2} \\
n_{1, t}=\delta n_{1, t-1}+\frac{1-\delta}{Z_{t-1}} \exp \left(\beta\left[-\left(x_{1, t-1}-(1+g) x_{3, t-1}+g x_{4, t-1}\right)^{2}+\eta U_{1, t-2}\right]\right) \\
x_{1, t}=\exp \left(-\frac{1}{200}\left|x_{1, t-1}\right|\right) \frac{1}{1+r}\left(\left[\delta n_{1, t-1}+\frac{1-\delta}{Z_{t-1}} \exp \left(\beta \left[-\left(x_{1, t-1}-(1+g) x_{3, t-1}+g x_{4, t-1}\right)^{2}+\right.\right.\right.\right. \\
\left.\left.\left.\eta U_{1, t-2}\right]\right)\right]\left((1+g) x_{1, t-1}-g x_{2, t-1}\right)+ \\
+\left[\delta n_{2, t-1}+\frac{1-\delta}{Z_{t-1}} \exp \left(\beta \left[-\left(x_{1, t-1}-(m+l) x_{3, t-1}+l x_{4, t-1}\right)^{2}+\right.\right.\right. \\
\left.\left.\left.\left.\eta U_{2, t-2}\right]\right)\right]\left((m+l) x_{1, t-1}-l x_{2, t-1}\right)\right) \\
x_{2, t}=x_{1, t-1} \\
x_{3, t}=x_{2, t-1} \\
x_{4, t}=x_{3, t-1}
\end{array}\right.
$$

It is straightforward to check that the fundamental steady state ${ }^{6}$ is a fixed point of the map, with $p_{t} \equiv p^{*}=p^{f}$ (i.e. $x_{1}=x_{2}=x_{3}=x_{4}=0$ ), the fraction of robot traders $n_{t} \equiv 0$ and the fractions of both heuristics $n_{1}^{*}=n_{2}^{*}=0.5$. We investigate its local stability. The Jacobian matrix $\mathbf{J}$ of the system at the steady state is given by

$$
J_{s}=\left[\begin{array}{ccccccc}
\eta & 0 & 0 & 0 & 0 & 0 & 0 \\
0 & \eta & 0 & 0 & 0 & 0 & 0 \\
\frac{\beta \eta(1-\delta)}{4} & -\frac{\beta \eta(1-\delta)}{4} & \delta & 0 & 0 & 0 & 0 \\
0 & 0 & 0 & \frac{(1+g+m+l)}{2(1+r)} & \frac{-g-l}{2(1+r)} & 0 & 0 \\
0 & 0 & 0 & 1 & 0 & 0 & 0 \\
0 & 0 & 0 & 0 & 1 & 0 & 0 \\
0 & 0 & 0 & 0 & 0 & 1 & 0
\end{array}\right]
$$

This Jacobian matrix has eigenvalues equal to 0 and $\eta$ (both of multiplicity 2 ), $\delta$ (of multiplicity 1) and the remaining two eigenvalues are the roots of the characteristic polynomial of the matrix

$$
J_{\lambda}=\left[\begin{array}{cc}
\frac{(1+g+m+l)}{2(1+r)} & \frac{-g-l}{2(1+r)} \\
1 & 0
\end{array}\right]
$$

\footnotetext{
${ }^{6}$ The fundamental steady state may not be the unique steady state of the model. In the parameter space we consider (that is the parameter values that are empirically relevant) an additional non fundamental steady state exists but it is always unstable.
} 
Since $\eta$ and $\delta$ are supposed to be smaller than 1, they do not determine a change in the stability of the system. We also assume $\delta \neq 1$, so that the impact of the heuristics varies over time and $\eta<1$ which means agents take into account their past performances. For these reasons the local stability conditions are completely determined by the eigenvalues of $J_{\lambda}$ and the coefficients of forecasting heuristics are the main driving forces. Nevertheless the parameters $\eta$ and $\delta$ affect the speed of convergence, being eigenvalues of the Jacobian matrix. Furthermore the local stability is not influenced by the intensity of choice $\beta$. Hence, the fundamental steady state of the asset pricing model is locally stable if all the eigenvalues of $J_{\lambda}$ lie inside the unit circle. When the heuristic coefficients are specified, the eigenvalues of $J_{\lambda}$ can be computed. Let

$$
\mathcal{P}(\lambda)=\lambda^{2}-\frac{1+g+m+l}{2(1+r)} \lambda+\frac{g+l}{2(1+r)}
$$

be the characteristic polynomial of $J_{\lambda}$. The stability region of the fixed point is determined by the following conditions (see e.g. Medio and Lines 2003):

$$
\left\{\begin{array}{l}
\mathcal{P}(1)=1-\operatorname{Tr}+\text { Det }>0 \\
\mathcal{P}(-1)=1+\operatorname{Tr}+\text { Det }>0 \\
\text { Det }<1
\end{array}\right.
$$

Figure 2 depicts the stability region in the parameter space $(m, g+l)$. The steady state of the switching model is locally stable when $\frac{g+l}{2}<R$, that is, when the average extrapolation coefficient is smaller than $R$. The fundamental steady state undergoes a Neimark-Sacker (NS henceforth) bifurcation, with complex eigenvalues on the unit circle, when $\frac{g+l}{2}=R^{7}$.

Moreover it is important to stress that in a nonlinear model, even if the fundamental steady state is locally stable, other attractors, such as a stable cycle or a quasi-periodic orbit, may co-exist. Hence the local stability properties of the fundamental steady state do not imply global stability.

\section{Model simulations and path dependence}

The heuristics switching mechanism matches individual forecasting behavior as well as aggregate market outcomes in the laboratory experiments. The simulations in Figure 3 show that the simple 2-type heuristics-switching model can replicate the three different aggregate market outcomes, i.e. monotonic convergence to the equilibrium, dampened oscillations and persistent oscillations. At the same time, the forecasts of the two heuristics are well coordinated (middle panels), consistent with the coordination of individual

\footnotetext{
${ }^{7} \mathrm{~A}$ straightforward computation shows that the NS-bifurcation is the only bifurcation that can destabilize the fundamental steady state, as eigenvalues +1 and -1 can not arise. See Gaunersdorfer et al. (2008) and Hommes (2013b) for a detailed discussion of Neimark-Sacker bifurcation in a similar asset pricing model with heterogeneous expectations.
} 


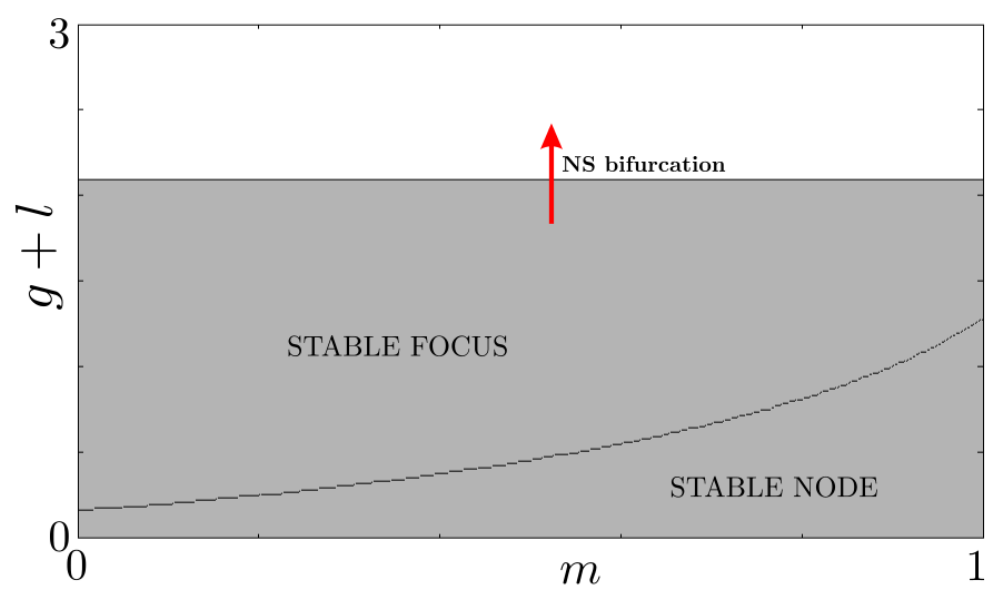

Figure 2: Local stability of the fundamental steady-state. Stability region (grey color) in the $(m, g+l)$ parameter space. The fundamental steady state $p^{f}$ becomes unstable through a Neimark-Sacker bifurcation along the horizontal line $g+l=2 R$.

expectations in the experiment (see Figure 1). The right panels in Figure 3 illustrate which of the two forecasting rules dominates in each of the three cases.

In all simulations in Figure 3 the anchor and adjustment rule is fixed, with $m=$ 0.5 and $l=1$, but the trend-rule has different trend-extrapolating coefficients. For a very weak trend-extrapolation coefficient $g=0.1$ (top panels) (almost) monotonic convergence to the fundamental steady state occurs, with the fractions of the two rules both approaching 50\%. For a somewhat larger trend-extrapolation coefficient $g=0.5$ oscillations around the fundamental price occur, through overshooting due to an initially dominating trend-following rule. These oscillations are dampened however, with the fractions of the two rules approaching $50 \%$ in the long run.

In the presence of strong trend-followers $(g=1.06)$ persistent oscillations around the fundamental price occur (Figure 3, bottom panels). The two forecasting rules are coordinated, consistent with experimental data, but the anchor and adjustment rule performs better than the trend-following rule especially around turning points. The fractions of the two rules also oscillate (with very small amplitude), with the anchor and adjustment rule clearly dominating, because it performs better along the price oscillations. In particular, because the anchor and adjustment rule uses a more cautious anchor (with more weight to the equilibrium level) it is able to predict turning points of the price oscillations much better than the pure trend-following rule which clearly overshoots (undershoots) at high (low) price levels and turning points. Our 2-type model thus explains 

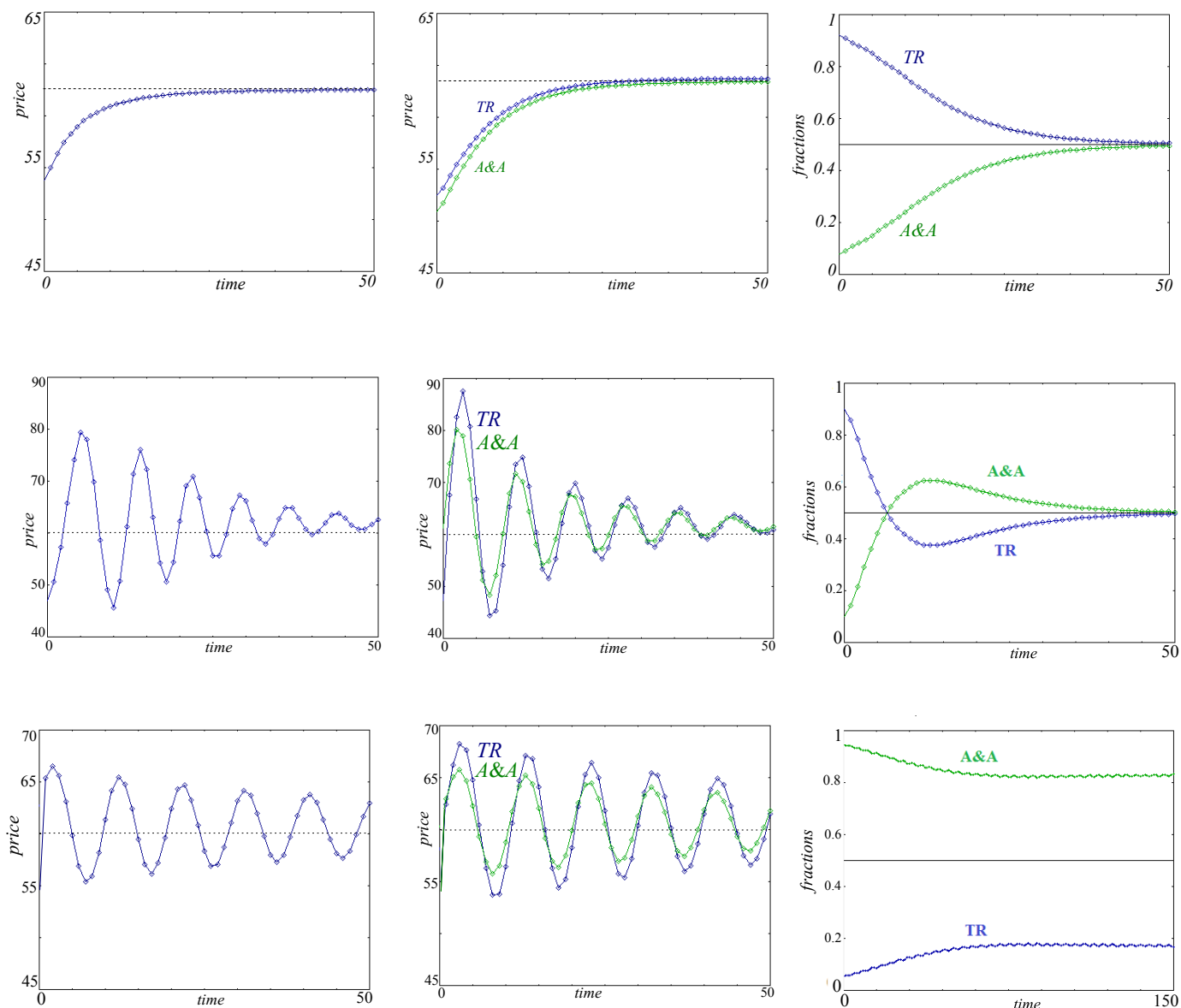

Figure 3: Coordination of forecasting heuristics in model simulations. Heuristic switching model simulations (Left), predictions of the two forecasting heuristics in the evolutionary switching model (Middle) and fractions of trend following (TR; blue) and anchoring and adjusting rule (A\&A; green) (Right). Benchmark parameters are $\beta=0.4, \eta=0.7, \delta=0.9$ and $m=0.5$. Almost monotonic convergence is obtained for $g=0.1, l=1$ (top panels), dampening oscillations for $g=0.5, l=1$ (central panels) and persistent fluctuations for $g=1.06, l=1$ (bottom panels). 
persistent oscillations through coordination of about $80 \%$ of the population on an almost self-fulfilling anchor and adjustment rule.

Figure 4 shows simulations of the 2-type heuristics switching model with all parameters of both forecasting rules fixed, but with different initial prices. The trend parameters $g=1.06$ and $l=1.035$ and the anchor parameter $m=0.5$ have been fixed close to empirically relevant and some of the individual estimated $\operatorname{AR}(2)$ forecasting rules in Hommes et al. (2005) and the 4-type switching model of Anufriev and Hommes (2012a). The 2-type heuristics switching model exhibits path dependence, with a locally stable fundamental steady state co-existing with stable (quasi-)periodic price oscillations. For an initial state $p_{0}=p_{1}=58.5$ prices converge to the fundamental steady state 60 (Figure 4, top panel LHS), with the fractions of the two forecasting rules almost constant and balanced, very close to $50 \%$ (top panel, RHS). For the initial state $p_{0}=p_{1}=52$ (bottom panel, LHS) prices exhibit persistent (quasi-)periodic oscillations, with timevarying fractions of the two forecasting rules (bottom panel, RHS). Along the price cycles the learning anchoring and adjusting heuristic performs better and dominates over the trend-following heuristic (bottom panel, RHS), but during the upswings and downswings the trend-following rule gets more followers thus sustaining and amplifying the price oscillations. Our simple 2-type heuristic switching model provides a stylized, behavioral explanation of path-dependent coordination on either a stable fundamental steady state or a stable periodic oscillation. In the presence of exogenous shocks the system may then switch between these different types of behavior.

These simulations illustrate path dependence in our model, a result that is established rigorously in Appendix A. To keep this analysis tractable we fix the parameters $\eta=$ $\delta=0$, so there is no memory in the performance measure and strategy updating is synchronous $^{8}$. Using advanced mathematical tools from bifurcation theory Appendix A shows rigorously that path-dependence with co-existence of a locally stable fundamental steady state and a stable periodic cycle is a generic feature of our simple 2-type behavioral heuristics switching model $^{9}$.

Figure 5 further illustrates the path-dependence by a 2-dimensional bifurcation diagram in the $(l, m)$ parameter plane. The vertical line $l=1.04$ denotes the NeimarkSacker (NS) bifurcation curve $l=2 R-g$; on the left (right) of this line, the fundamental steady state is locally stable (unstable). Different colors in Figure 5 refer to stable periodic cycles of different periods. The purple region corresponds to parameter combinations $(l, m)$ for which a stable 10 cycle exists. This is remarkably close to the period 10-12 of persistent oscillations in the lab experiments (see Figure 1b). The region of the stable 10-cycle extends to the left of the NS bifurcation curve (the vertical line $l=1.04$ ), showing co-existence of a locally stable fundamental steady state and a (locally) stable period 10 cycle. The point $A$ in Figure 5 corresponds to the simulations of Figure 4 with

\footnotetext{
${ }^{8}$ For $\eta=\delta=0$ the dimension of the system is reduced from 7 to 4 .

${ }^{9}$ Gaunersdorfer et al. (2008) consider a similar 2-type asset pricing model with fundamentalists versus trend-followers exhibiting such path-dependence and use the model to explain clustered volatility, that is, irregular switching between a quiet phase with prices close to fundamental and a more volatile phase with large price oscillations. Heterogeneity and strategy switching thus explains clustered volatility (cf. e.g. Lux and Marchesi, 1999).
} 

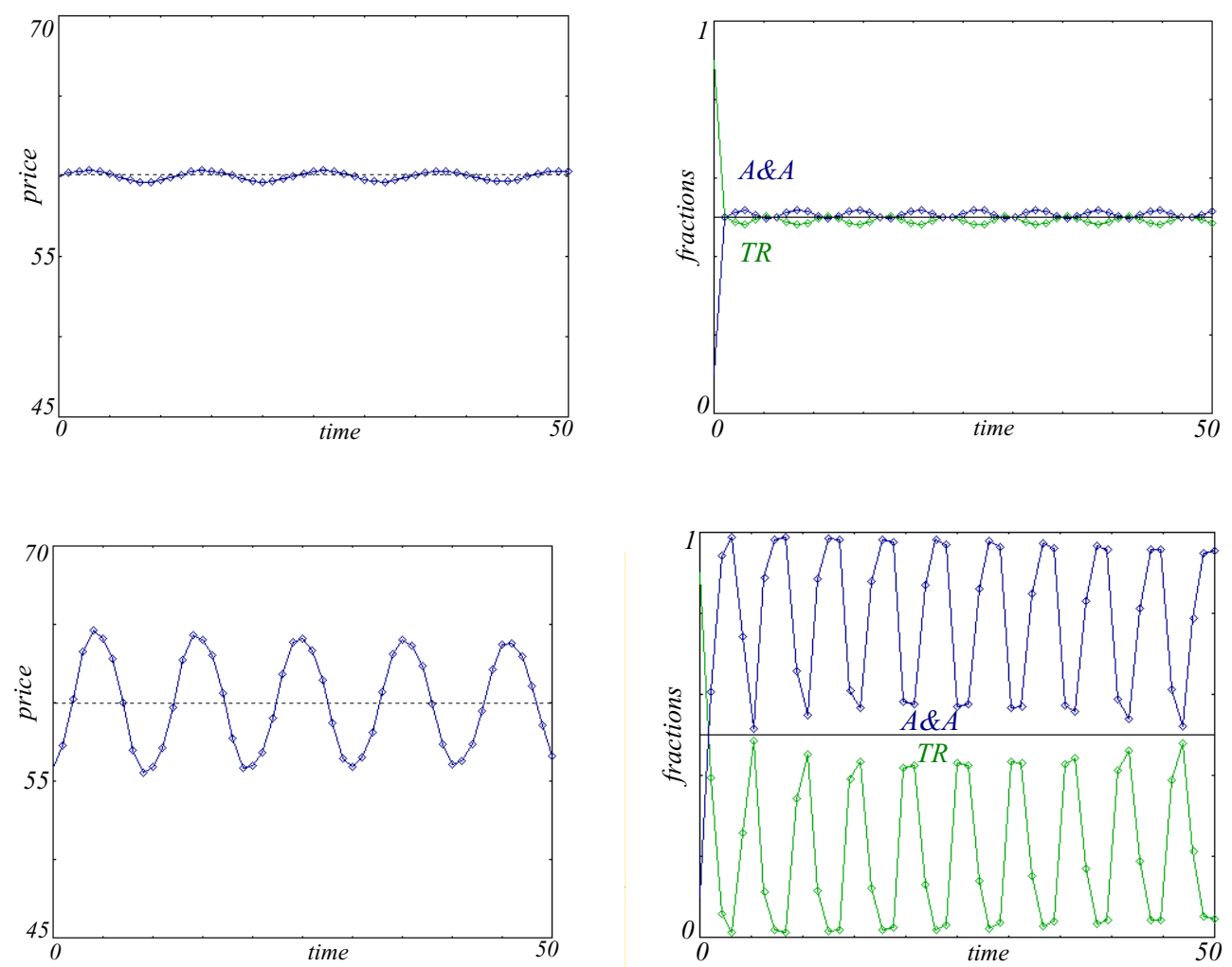

Figure 4: Path dependence in heuristic switching model. Prices (left) for the 2-type heuristics switching model and fractions of the two forecasting heuristics (right), the trend following (TR, blue lines) and the learning anchoring and adjusting (A\&A, green lines) rules. The heuristics switching model exhibits path dependence for parameter values $g=1.06, l=$ $1.035, m=0.5, \beta=0.4$ and $\eta=\delta=0$, with a locally stable fundamental steady state co-existing with stable (quasi-)periodic price oscillations. For the initial state $p_{0}=p_{1}=58.5$ prices convergence to the fundamental steady state 60 (top panel, LHS), with the fractions of the two forecasting rules almost constant and balanced, very close to 50\% (top panel, RHS). For the initial state $p_{0}=p_{1}=52$ (bottom panel, LHS) prices exhibit persistent (quasi-)periodic oscillations, with time-varying fractions of the two forecasting rules, but the learning anchoring and adjusting heuristic dominating over the trend-following heuristic (bottom panel, RHS). Since $\eta=\delta=0$, i.e. no memory and synchronous updating, the fluctuations in fractions are much faster than in the simulations of Figure 3. 
$l=1.035$ and $g=1.06$ and it lies in the path dependence region where the locally stable fundamental steady state coexists with an attracting closed curve with stable (quasi)periodic behavior. The point on the vertical line $l=1.04$ labeled $m^{*}$ is a so-called Chenciner bifurcation point and plays an important role in the mathematical analysis proving generic existence of path-dependence in the heuristics switching model (see the mathematical appendix A).

When buffeted with noise, our model with co-existing attractors can generate volatility clustering, as illustrated in Figure 6. In particular a noise term (normal distribution, with standard deviation $\sigma=0.1$ ) has been added to the equilibrium pricing equation of the map (13). Notice that this is equivalent to adding a small fraction of noise traders operating into the market. Due to the presence of the noise traders, the market switches irregularly between a low volatility phase, with prices close to the fundamental value, and a high volatility phase, with prices exhibiting temporary departures from the fundamental steady state, triggered by noise traders and amplified by trend-followers.

Finally, the bifurcation diagrams in Figure 7, shows the abrupt change in the dynamics of the model when the subcritical NS bifurcation occurs (Figure 7a) and when the initial values exit the basin of attraction of the steady state (Figure $7 \mathrm{~b}$ ). Indeed when the subcritical NS bifurcation occurs and the fundamental price becomes unstable, the trajectories starting close to the equilibrium suddenly exhibit quite large fluctuations, as Figure 7a reports. But even when $p^{f}$ is stable, endogenous fluctuations are a possible outcome of the model (see $7 \mathrm{~b}$ ), since approaching the bifurcation value the basin of attraction of the fundamental price becomes smaller and smaller and the price may exit from it, due to some shocks. Comparing the two bifurcations diagrams of Figure 7, we can also observe that different abrupt changes of the price dynamics exist when the parameter $l$ increases. In particular we may have an increase in the amplitude of the oscillations as well as transition from quasi-periodic trajectories to periodic ones. These changes are due to new multistability windows (for instance, at $l=1.048$ two attracting closed curves coexist and at $l=1.064$ an attracting closed curve coexists with a stable cycle of period 9) and confirm that the strong nonlinearity of the model implies very complex dynamical behaviors. The analysis of these latter dynamic configurations is left to further research, being beyond the aim of the present paper. 


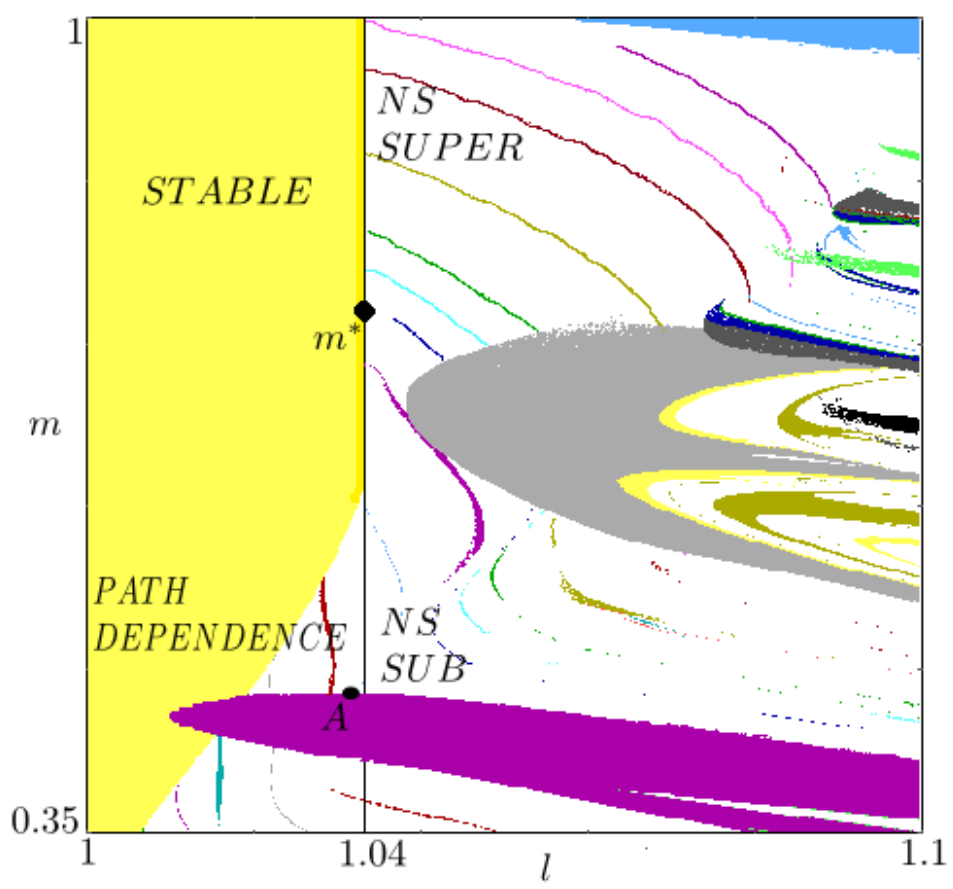

Figure 5: Bifurcation diagram in the $(l, m)$ parameter plane showing path dependence. Other parameters are fixed at $g=1.06, \delta=0, \eta=0$ and $\beta=0.4$. The vertical line $l=1.04$ denotes the Neimark-Sacker (NS) bifurcation curve $l=2 R-g$. On the left (right) of this line, the fundamental steady state is locally stable (unstable). Different colors refer to stable periodic cycles of different periods. The point $A$ corresponds to the simulations of Figure 4 with $l=1.035, m=0.5$ and $g=1.06$ and lies in the path dependence region where the locally stable fundamental steady state coexists with an attracting closed curve with stable (quasi-)periodic behavior. When the vertical line is crossed to the right the fundamental steady state is destabilized either via a supercritical (for $m>m^{*}$ ) or subcritical (for $m<m^{*}$ ) NS bifurcation. The point labeled $m^{*}$ is a Chenciner bifurcation point and proves the existence of a path-dependence region (see the Appendix). 


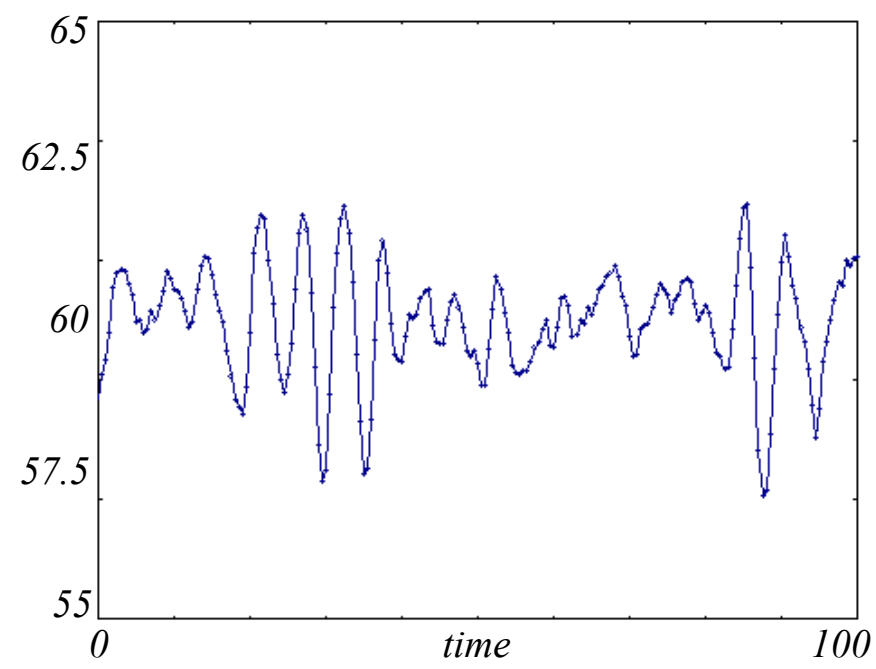

Figure 6: Time series of prices for the model with small noise. The model with coexistence of a stable fundamental steady state and a (quasi-)periodic attractor exhibits clustered volatility. Parameter values are: $l=1.035, m=0.5, g=1.06, \beta=0.4, \delta=0, \eta=0$.

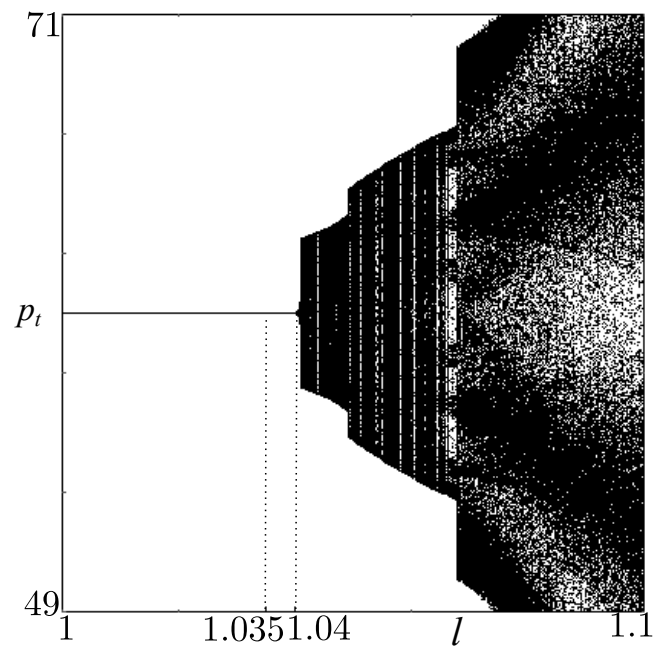

(a) Initial prices: $p_{0}=59.9, p_{1}=59.9$

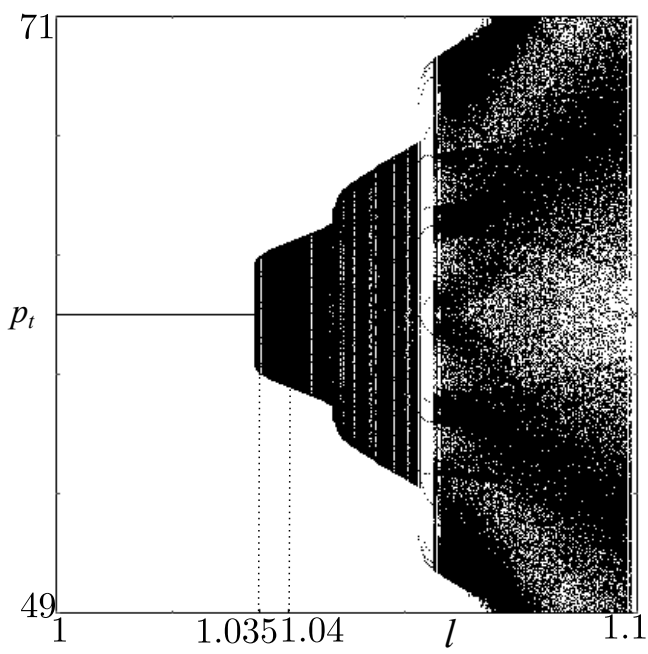

(b) Initial prices: $p_{0}=50, p_{1}=50$

Figure 7: 1D bifurcation diagram with respect to $l$. Other parameters are fixed at $g=1.06, m=0.5, \delta=0, \eta=0, \beta=0.4$. In the left panel when the subcritical Neimark-Sacker bifurcation occurs $(l=1.04)$ the price suddenly jumps to quasi-periodic trajectories. The quasiperiodic trajectories appear at a lower value of $l$ and coexist with the stable equilibrium price (right panel). The parameter value $l=1.035$ corresponds to Figure 5 . 


\section{Concluding Remarks}

Traditional finance assumes that all agents have rational expectations. On the other hand laboratory experiments with human subjects have shown that agents do not forecast rationally, but follow simple forecasting heuristics (rules of thumb) that may sometimes lead them to the rational fundamental benchmark and at other times agents may coordinate on destabilizing trend-following strategies leading to recurrent market bubbles and crashes. We have presented a simple behavioral heuristics switching model explaining path dependent coordination of individual forecasting as well as aggregate market behavior.

The model is made up with two simple but different forecasting rules, each of which can generate its own type of dynamics. In every period the forecasting strategy is selected among the population of heuristics and agents adapt their selection over time, based on the relative performance of the heuristics. This evolutionary selection mechanism exhibits path dependence and may either enforce convergence to the fundamental price, with the two strategies balanced, close to $50 \%$, or it may lead to persistent price oscillations around the fundamental value with the fractions of both strategies evolving continuously over time. Similar price oscillations are characteristic of real markets and our model thus provides, at least qualitatively, an explanation of stylized facts, such as volatility clustering -switching between calm and turbulent market phases- observed in real financial markets.

What drives these results? A key feature is the positive feedback in the system and the existence of almost self-fulfilling equilibria. Positive feedback is characteristic of near-unit root systems and -because the discount factor $1 /(1+r)$ is indeed very close to 1 - the price generating equation (1) follows a near-unit root process. What the lab experiments in Hommes et al. (2005) then have shown is that agents may coordinate on an oscillatory pattern of almost self-fulfilling equilibria. Our simple 2-type model shows that such patterns can be the path dependent outcomes of a simple evolutionary learning process selecting among trend-following and anchor and adjustment heuristics. Our results also provide some evidence of the empirical relevance of the Chenciner bifurcation to explain observed path-dependence in laboratory markets with near unit root positive feedback. It may be interesting for future work to study path-dependent behavior in laboratory experiments with occasional large shocks to fundamentals (see Bao et al. (2012) for how large shocks may affect coordination in positive versus negative feedback experimental markets).

If the evolutionary interaction of boundedly rational agents with different trading strategies extols volatility, there are important consequences for regulatory policy in financial markets. Good or bad news in the markets can be amplified by the evolutionary mechanism. Since we are embedded in an increasingly globalized financial world, small changes in fundamentals in one country may generate changes in asset prices of other countries. Policy may avoid coordination on almost self-fulfilling oscillations by transparency about market fundamentals promoting coordination on underlying fundamentals. Another stabilizing policy could be to weaken the positive feedback in the 
financial system - e.g. through increasing the risk free interest rate and thus moving the discount factor away from unit root- in order to make coordination on almost selffulfilling equilibria less likely. More behavioral models are needed in order to address the important question of how policy could stabilize the system and avoid coordination on non-rational, but almost self-fulfilling aggregate outcomes. 


\section{Appendix}

\section{A Mathematical Appendix on Path Dependence}

With fixed parameter values, the 2-type model can explain path dependent coordination on either a locally stable fundamental steady state or an attracting closed invariant curve for different initial states, as illustrated in Figure 4. In this mathematical appendix we show analytically that path dependence is a generic feature of our nonlinear behavioral model. The main argument relies on the occurrence of a so-called degenerate NeimarkSacker or Chenciner bifurcation in the model, implying path dependence due to the existence of a region in the parameter space where a local stable steady state and an attracting invariant curve with (quasi-)periodic dynamics co-exist. Advanced bifurcation tools may not be very familiar to economists, but we stress that a Chenciner bifurcation is not special at all, but rather a generic phenomenon of nonlinear systems with two or more parameters. There are some papers in the economic literature that study the effects of a Chenciner bifurcation, e.g. Neugart and Tuinstra (2003) and Lines and Westerhoff (2010, 2012)

In this appendix we sketch the main mathematical arguments for path-dependence. Kuznetsov (2004) presents a detailed mathematical overview of advanced bifurcation theory, including the Chenciner and other generic bifurcations of nonlinear systems.

For analytical tractability, we restrict our analysis to the case of no memory in the performance measure $(\eta=0)$ and synchronous strategy updating $(\delta=0)$, so that the dimension of the system in Section 4 reduces from 7 to 4 . The parameter space is

$$
P=\{(\beta, g, m, l, R): \beta>0, g>0,0<m<1, l>0, R>1\}
$$

Recall from Section 4 that the fundamental steady state may become unstable due to a Neimark-Sacker bifurcation at $g+l=2 R$. Generally a steady state loses stability through a Neimark-Sacker (NS henceforth) bifurcation when its Jacobian matrix has two complex eigenvalues lying on the unit circle with all other eigenvalues inside the unit circle. Two kind of NS bifurcations can be distinguished:

- supercritical when, immediately after the bifurcation, the unstable steady state is surrounded by an attracting closed curve corresponding to periodic or quasiperiodic dynamics;

- subcritical when, immediately before the bifurcation, the stable equilibrium is surrounded by a repelling closed curve which shrinks and at the bifurcation merges with the fixed point leaving a repelling focus.

Which NS bifurcation, supercritical or subcritical, occurs depends on the sign of the socalled first Lyapunov coefficient of the normal form. When the first Lyapunov coefficient vanishes, i.e. equals 0 , a degenerate NS or Chenciner bifurcation occurs. The Chenciner bifurcation is a generic phenomenon in two or higher dimensional nonlinear systems with two or more parameters. More precisely, non-degenerate NS bifurcations are generic in 
two or higher dimensional nonlinear systems with a single parameter. For two-parameter systems one generically can find a pair of parameters for which the Lyapunov coefficient in the normal form of the NS bifurcation vanishes and the NS bifurcation then becomes degenerate (i.e. a Chenciner bifurcation).

In the 2-D bifurcation diagram of our 2-type switching model in Figure 5 the Chenciner bifurcation point is labeled $m^{*}$, lying on the NS-bifurcation curve (the vertical line $l=1.04$, with $g$ fixed at $g=1.06)$. Bifurcation theory implies that close to a Chenciner bifurcation point, there exists a path dependence region in the parameter space, where a locally stable steady state co-exists with an attracting invariant curve. Figure 5 shows the path dependence region in the lower left corner, where e.g. part of the purple region to the left of the NS-bifurcation vertical line $l=1.04$ shows co-existence of a locally stable steady state and a stable 10-cycle. The point labeled $A$ in Figure 5 corresponds to our simulations of path dependence in Figure 4.

We further study the locus of the degenerate NS (Chenciner) bifurcation points in the parameter plane in our 2-type switching model. As we said above the NS bifurcation manifold is given by

$$
H=\left\{(\beta, g, m, l, R) \in P: \frac{g+l}{2}=R\right\} .
$$

Figure 8 shows the locus of the Chenciner bifurcation points in the $(m, g)$ parameter plane. The vertical axis plots the parameter $g$ (black) and $l$ (grey), with $g+l=2 R$ so that each point is a NS bifurcation point. The horizontal axis plots the parameter $m$. The figure illustrates for which pair of parameters $(m, g)$ the NS bifurcation is subcritical (SUB) or supercritical (SUPER). The curve in Figure 8 corresponds exactly to the points where the NS bifurcation is degenerate, i.e. a Chenciner bifurcation. The point on the curve for $m=m^{*} \approx 0.747$ corresponds to the Chenciner bifurcation point labeled $m^{*}$ in Figure 5. The figure shows that for relatively small $g$ values the steady state loses stability through a subcritical NS bifurcation. When $g$ is sufficiently large the NS bifurcation can be either subcritical or supercritical, the latter arising as $m$ increases.

The sharp and sudden change in the dynamical behavior of the system associated with the subcritical NS bifurcation may be furthermore accompanied by a so-called crater bifurcation (see Kuznetsov (2004, pp.418-422); Kind (1999) and Agliari et al. $(2005,2006))$ : two invariant closed curves, one repelling and one attracting, appear surrounding the fixed point when it is still stable. This occurrence is related to the concept of corridor stability as developed by Leijonhufvud (1973). Large shocks do not lead to a totally unstable dynamic but result in permanent, wide and bounded oscillations, and can be observed in the present model after the occurrence of the NS bifurcation. 


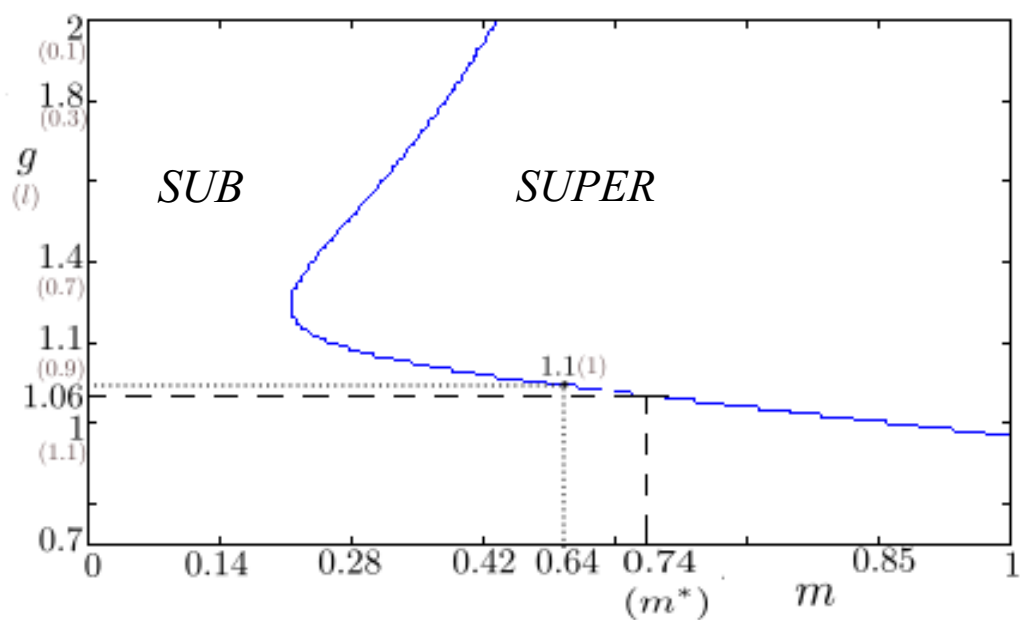

Figure 8: Plot of the locus of degenerate NS (Chenciner) bifurcation points. The curve marks the transition from a subcritical $(S U B)$ to a supercritical (SUPER) bifurcation. On the horizontal axis we have reported $m$ values, while on the vertical axis both $g$ (black color) and $l$ (gray color) values are displayed, such that $g+l=2 R$ (i.e. the parameter are at NS bifurcation values). Other parameter values are: $\delta=0, \eta=0, \beta=0.4$ and $R=1.05$. Points on the curve are the Chenciner bifurcation points: for empirically relevant parameters $g$ (and $l$ ), it is possible to compute the corresponding Chenciner point $m^{*}$ that delimits the path dependence region. 


\section{References}

[1] A. Agliari, G.I. Bischi, R. Dieci, L. Gardini. Global bifurcations of closed invariant curves in two-dimensional maps: A computer assisted study. International Journal of Bifurcations and Chaos, No. 15, pp. 1285-1328, 2005.

[2] A. Agliari, G. I. Bischi, L. Gardini. Some methods for the global analysis of closed invariant curves in two-dimensional maps. In Business Cycle Dynamics (pp. 7-47). Springer Berlin Heidelberg, 2006.

[3] M. Anufriev, C.H. Hommes. Evolutionary Selection of Individual Expectations and Aggregate Outcomes in Asset Pricing Experiments. American Economic Journal: Microeconomics, Vol.4 (4), pp. 35-64, 2012a.

[4] M. Anufriev, C.H. Hommes. Evolution of Market Heuristics. Knowledge Engineering Review, Vol.27 (2), pp. 255-271, 2012b.

[5] W. Arthur. Economic Agents that Behave like Human Agents. Journal of Evolutionary Economics, No. 3, pp. 1-22, 1993.

[6] Bao, T., Hommes, C., Sonnemans, J., and Tuinstra, J. (2012). Individual expectations, limited rationality and aggregate outcomes. Journal of Economic Dynamics and Control, 36(8), 1101-1120.

[7] T. Bao, C.H. Hommes and T. Makarewicz. Bubble Formation and (In)Efficient Markets in Learning-to-Forecast and-Optimise Experiments, The Economic Journal, forthcoming 2016.

[8] N. Barberis and R. Thaler. A survey of behavioral finance, Handbook of the Economics of Finance, Edited by G.M. Constantinidis, M. Harris and R. Stulz, Elsevier, pp.1051-1121, 2003.

[9] W.A. Brock, C.H. Hommes. A Rational Route to Randomness. Econometrica, No. 54, pp. 1059-1095, 1997.

[10] W.A. Brock, C.H. Hommes. Heterogeneous Beliefs and Routes to Chaos in a Simple Asset Price Model. Journal of Economic Dynamics and Control, No. 22, pp. 12351274, 1998.

[11] J.B. DeLong, A. Shleifer, L.H. Summers and R.J. Waldmann. Noise trader risk in financial markets, Journal of Political Economy 98, 703-738, 1990.

[12] C. Diks, R. Van Der Weide. Herding, asynchronous updating and heterogeneity in memory in a CBS. Journal of Economic Dynamics and Control, No. 29, pp. 741-763, 2005.

[13] M. Dufwenberg, Banking on Experiments, Working Paper Bocconi University, November 2014. 
[14] J.D. Farmer, D. Foley. The economy needs Agent-Based modelling. Nature, Vol. 460, No. 7256, pp. 685-685, 2009.

[15] A. Gaunersdorfer, C.H. Hommes, F.O.O. Wagener. Bifurcation routes to volatility clustering under evolutionary learning. Journal of Economic Behavior and Organization, vol. 67(1), pp. 27-47, 2008.

[16] P. Heemeijer, C.H. Hommes, J. Sonnemans, J. Tuinstra. Price stability and volatility in markets with positive and negative expectations feedback: An experimental investigation. Journal of Economic Dynamics and Control, 33(5), 1052-1072, 2009.

[17] C.H. Hommes. Heterogeneous agent models in economics and finance. in Handbook of Computational Economics, Vol. 2, chapter 23, pp. 1009-1186, 2006.

[18] C.H. Hommes. (2013a) Reflexivity, expectations feedback and almost self-fulfilling equilibria: economic theory, empirical evidence and laboratory experiments. Journal of Economic Methodology 20:4, 406-419.

[19] C.H. Hommes. Behavioral Rationality and Heterogeneous expectations in Complex Economic Systems. Cambridge University Press, 2013b.

[20] C.H. Hommes, J. Sonnemans, J. Tuinstra, H. van de Velden. Coordination of expectations in asset pricing experiments. The Review of Financial Studies, 18(3), pp. 955-980, 2005.

[21] C.H. Hommes, J. Sonnemans, J. Tuinstra, H. van de Velden. Expectations and bubbles in asset pricing experiments. Journal of Economic Behavior and Organization, 67(1), pp. 116-133, 2008.

[22] Huber, J., \& Kirchler, M. (2012). The impact of instructions and procedure on reducing confusion and bubbles in experimental asset markets. Experimental Economics, 15(1), 89-105.

[23] D. Kahneman, A. Tversky. On the Psychology of Predictions. Psychological Review, No. 80, 237-251, 1973.

[24] C. Kind. Remarks on the economic interpretation of Hopf bifurcations. Economics Letters, 62(2), pp. 147-154, 1999.

[25] C. Kindleberger. Manias, Panics 8 Crashes: a History of Financial Crisis. Wiley, 2001.

[26] Kirchler, M., Huber, J. and Thomas StÃ $₫$ ckl. (2012). Thar She Bursts: Reducing Confusion Reduces Bubbles. American Economic Review, 102(2): 865-83.

[27] Y.A. Kuznetsov. Elements of Applied Bifurcation Theory. Springer (3rd ed.): New York, 2004. 
[28] B. LeBaron. Agent-Based computational finance. Handbook of Computational Economics, Vol. 2, chapter 24, pp. 1187-1233, 2006.

[29] A. Leijonhufvud. Effective Demand Failures. The Swedish Journal of Economics, Vol. 75, No. 1, Stabilization Policy, pp. 27-48, 1973.

[30] Lines, M., \& Westerhoff, F. (2010). Inflation expectations and macroeconomic dynamics: the case of rational versus extrapolative expectations. Journal of Economic Dynamics and Control, 34(2), 246-257.

[31] Lines, M., \& Westerhoff, F. (2012). Effects of inflation expectations on macroeconomic dynamics: extrapolative versus regressive expectations. Studies in Nonlinear Dynamics \& Econometrics, 16(4).

[32] T. Lux, M. Marchesi. Volatility clustering in financial markets: a micro-simulation of interacting agents. International Journal of Theoretical and Applied Finance, No. 3, pp. 675-702, 2000.

[33] A. Medio, M. Lines. Nonlinear dynamics. A primer. Cambridge University Press, 2003.

[34] Neugart, M., \& Tuinstra, J. (2003). Endogenous fluctuations in the demand for education. Journal of Evolutionary Economics, 13(1), 29-51.

[35] S. Palan, A Review of Bubbles and Crashes in Experimental Asset Markets, Journal of Economic Surveys 27, 570-588, 2013.

[36] R. Shiller. Irrational Exuberance. Princeton University Press, 2000.

[37] V.L. Smith, G.L. Suchanek and A.W. Williams, 1988, Bubbles, Crashes and Endogenous Expectations in Experimental Spot Asset Markets, Econometrica, 56, 1119-1151.

[38] A. Tversky, D. Kahneman. Judgement under uncertainty: Heuristic and Biases. Science, Vol. 185, No. 4157, pp. 1124-1130, 1974. 